\title{
Liuwei Dihuang, a traditional Chinese medicinal formula, inhibits proliferation and migration of vascular smooth muscle cells via modulation of estrogen receptors
}

\author{
YAYUN ZHANG $^{1 *}$, XING QIAN $^{1 *}$, XIN SUN $^{1}$, CHAO LIN $^{1}$, YI JING $^{1}$, YUAN YAO $^{1}$, ZHI MA $^{1}$, MEIYU KUAI $^{1}$,

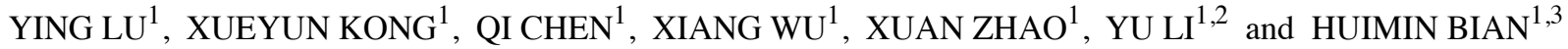

Schools of ${ }^{1}$ Pharmacy and ${ }^{2}$ Medicine and Life Sciences; ${ }^{3}$ Jiangsu Key Laboratory for Pharmacology and Safety Evaluation of Chinese Materia Medica, Nanjing University of Chinese Medicine, Nanjing, Jiangsu 210023, P.R. China

Received January 25, 2016; Accepted November 10, 2017

DOI: $10.3892 /$ ijmm.2018.3622

\begin{abstract}
The phenotypic modulation of vascular smooth muscle cells (VSMCs) serves an important role in atherosclerosis-induced vascular alterations, including vascular remodeling. However, the precise mechanisms underlying VSMC phenotypic modulation remain to be elucidated. Our previous study demonstrated that Liuwei Dihuang formula (LWDHF) could improve menopausal atherosclerosis by upregulating the expression of estrogen receptors (ERs). The present study examined the role of ERs in the effects of LWDHF on VSMC phenotypic modulation. VSMC proliferation and cell cycle progression were examined by MTT assay and flow cytometry, respectively. The expression levels of $\alpha$-smooth muscle actin, osteopontin and ERs were determined using reverse transcription-quantitative polymerase chain reaction (RT-qPCR) and western blot analysis. Cell ultrastructure was observed under an electron microscope. F-actin polymerization was detected by fluorescein isothiocyanate-phalloidin staining using fluorescence microscopy. A modified Boyden chamber assay was employed to assess VSMCs migration. Small interfering (si)RNA technology was used to examine the role of $\mathrm{ER} \alpha$ in the effects of LWDHF on phenotypic modulation. The results indicated that LWDHF (3-12 $\mu \mathrm{g} / \mathrm{ml})$ inhibited proliferation and induced a cell cycle arrest in VSMCs treated with angiotensin II (Ang II; $100 \mathrm{nM}$ ) in a concentration-dependent manner. In addition, Ang II-stimulated migration of VSMCs and reorganization of actin were markedly inhibited by treatment with $12 \mu \mathrm{g} / \mathrm{ml}$ LWDHF. Results of RT-qPCR and western blotting demonstrated that LWDHF markedly stimulated tran-
\end{abstract}

Correspondence to: Professor Huimin Bian or Professor $\mathrm{Yu} \mathrm{Li}$, School of Pharmacy, Nanjing University of Chinese Medicine, 138 Xianlin Avenue, Qixia, Nanjing, Jiangsu 210023, P.R. China

E-mail: hmbian@sina.com

E-mail: qxliyu@sina.com

*Contributed equally

Key words: Liuwei Dihuang formula, vascular smooth muscle cell, proliferation, migration, phenotypic switching, estrogen receptor, angiotensin II scription and expression of ER $\alpha$ and ER $\beta$, and inhibited VSMC synthetic phenotype. Furthermore, LWDHF-induced inhibition of phenotypic switching was partially suppressed by tamoxifen, and transfection with ER $\alpha$ siRNA markedly abolished the effects of LWDHF on VSMC phenotypic switching. In conclusion, these results revealed that ER $\alpha$ served an important role in LWDHF-induced regulation of the VSMC phenotype, including proliferation and migration.

\section{Introduction}

Abnormal proliferation of vascular smooth muscle cells (VSMCs) is believed to serve an important role in the formation of atherosclerotic plaques and restenosis following percutaneous coronary intervention $(1,2)$. Vascular remodeling has recently garnered increasing attention due to its critical role in the progression of vascular occlusion diseases (3). During vascular lesion formation, phenotypic switching of VSMCs from the physiological contractile phenotype to the pathophysiological synthetic phenotype occurs, and VSMCs migrate to the intima (4). Under normal conditions, VSMCs constitute the major structural component of the vasculature, and are crucial for maintaining vessel tone, blood pressure and blood flow. In the media layer of mature blood vessels, VSMCs exhibit a differentiated and contractile phenotype, characterized by the expression of contractile proteins, including $\alpha$-smooth muscle actin ( $\alpha$-SMA), calponin and smooth muscle heavy chain (5). In various vascular pathologies, VSMCs undergo a rapid and reversible alteration from a quiescent contractile phenotype to a proliferative and secretory phenotype, which is characterized by increased proliferation, migration and extracellular matrix (ECM) production (6), and osteopontin (OPN) is the main protein marker of synthetic phenotypes. The synthetic phenotype of VSMCs acts as a critical factor in various cardiovascular diseases, including atherosclerosis, restenosis after angioplasty or bypass, and hypertension $(7,8)$.

It is well known that numerous cytokines and growth factors are released to stimulate VSMC proliferation during vascular injury repair $(9,10)$. Angiotensin II (Ang II), which has previously been reported to serve an important role in normal vascular physiology and cardiovascular disease, is a 
biologically active peptide of the renin-angiotensin system, which modulates vascular tone and structure. Ang II interacts with the type 1 receptor on SMCs and promotes a switch from a contractile to a synthetic phenotype of VSMCs, leading to changes in the contractile machinery and VSMC proliferation $(11,12)$. In addition, Ang II may promote the production of various growth-stimulating factors, including fibroblast growth factor, transforming growth factor- $\beta$, insulin-like growth factor and platelet-derived growth factor, leading to proliferation of VSMCs, as well as migration of VSMCs to the intima, thus contributing to the formation of atherosclerosis.

Liuwei Dihuang (LWDH) is a traditional Chinese herbal formulation that has been used for $>1,000$ years in China. According to traditional Chinese medicine, LWDH formula(LWDHF) has the ability to tonify kidney 'Yin', and can be used clinically to treat hypertension, diabetes and numerous diseases associated with 'Yin' deficiency in the kidney of perimenopausal and postmenopausal women. LWDHF is comprised of six Chinese herbs: Shu Di Huang (Radix Rehmanniae Preparata), Shan Zhu Yu (Fructus Corni), Shan Yao (Rhizoma Dioscoreae), Ze Xie (Rhizoma Alismatis), Mu Dan Pi (Cortex Moutan) and Fu Ling (Poria). LWDHF is orally administered as a decoction, or in pill form, for the treatment of aging-associated functional decline and geriatric diseases, particularly for diabetes, cognition and memory decline in China and Japan $(13,14)$. Recently, numerous studies have revealed the beneficial effects of LWDHF on the cardiovascular system in postmenopausal women. Our previous study demonstrated that LWDHF exerted preventive and therapeutic effects on atherosclerosis in ovariectomized rats $(15,16)$, and that $L W D H F-$ medicated serum protected human umbilical vein endothelial cells (HUVECs) against hydrogen peroxide $\left(\mathrm{H}_{2} \mathrm{O}_{2}\right)$-induced apoptosis and upregulated the expression of estrogen receptors (ERs) following vascular endothelial cell injury in vitro (16). A significant increase in the incidence and mortality of cardiovascular diseases has been noted in postmenopausal women, thus indicating that estrogen may have an important protective effect on the vasculature (17). Furthermore, numerous studies demonstrated that estrogen could lower the risk of cardiovascular disease in women (18) and inhibit VSMC proliferation following injury (19-21). Therefore, the present study aimed to investigate the effects of LWDHF on Ang II-induced VSMC proliferation and migration, and to explore the role of ERs in the effects of LWDHF. To the best of our knowledge, the present study is the first to demonstrate the suppressive effects of LWDHF on Ang II-induced proliferation and migration of VSMCs. In addition, the molecular mechanism by which LWDHF inhibits proliferation and migration may be associated with modulating the phenotypic modulation of VSMCs, which was partially mediated by the ER $\alpha$-activated estrogen signaling pathway. Understanding the cellular and molecular pathways of LWDHF may result in the identification of novel therapeutic strategies for the treatment of atherosclerosis and restenosis in perimenopausal or postmenopausal women.

\section{Materials and methods}

Reagents and antibodies. Human Ang II, tamoxifen and cell proliferation reagent 3-(4,5-dimethylthiazol-2-yl)-2,5-di- phenyltetrazolium bromide (MTT) were purchased from Sigma-Aldrich (Merck KGaA, Darmstadt, Germany). Antibodies used to detect the protein expression levels of $\alpha$-SMA (ab124964), OPN (ab8448), ER $\beta$ (ab92306), $\beta$-actin and $\beta$-tubulin were obtained from Abcam (Cambridge, MA, USA). Anti-ER $\alpha$ antibody was purchased from Cell Signaling Technology, Inc.(Danvers, MA, USA). ER $\alpha$ small interfering (si)RNA and control siRNA were purchased from Santa Cruz Biotechnology, Inc. (Dallas. TX, USA).

Preparation of LWDH. The method of LWDH preparation was reported by Yang et al (22). Briefly, Rehmannia glutinosa Libosch. (Scrophulariaceae family), Cornus officinalis Sieb. (Cornaceae family), Dioscorea $o$ pposita Thunb. (Dioscoreaceae family), Alisma orientale (G. Samuelsson) Juz (Alismataceae family), Poria cocos (Schw.) Wolf (Polyporaceae family) and Paeonia suffruticosa Andrews (Paeoniaceae family) were mixed at a ratio of $8: 4: 4: 3: 3: 3$. The mixture was twice decocted in distilled water for $30 \mathrm{~min}$. The water extracts were concentrated to $2 \mathrm{~g} / \mathrm{ml}$ for further use. High-performance liquid chromatography was used to analyze the constituents of LWDH (22). Five major constituents, including gallic acid, paeonoside, verbascoside, loganin and paeoniflorin, were identified in LWDHF (Fig. 1). Their contents were $2.74,0.05,0.06,0.74$ and $0.43 \mathrm{mg} / \mathrm{g}$, respectively. The standard samples of gallic acid, paeonoside, verbascoside, loganin and paeoniflorin were purchased from Beijing Beina Chuanglian Biotechnology Research Institute (Beijing, China).

Cell culture. Primary VSMCs were isolated from thoracic aortas of 7-week-old male Sprague-Dawley rats by explant technique, and were then cultured in Dulbecco's modified Eagle's medium (DMEM) supplemented with $10 \%$ fetal bovine serum (FBS), $100 \mathrm{kU} / 1$ benzylpenicillin and $100 \mathrm{mg} / \mathrm{l}$ streptomycin at $37^{\circ} \mathrm{C}$ in a humidified chamber containing $5 \% \mathrm{CO}_{2}$ (23). The identification of VSMCs was performed by $\alpha$-SMA immunostaining; $>90 \%$ of cells were $\alpha$-SMA-positive and exhibited a spindle-shaped appearance. VSMCs were passaged by trypsinization, and cells at passages 3-7 were used for subsequent experiments to ensure genetic stability of the culture. All animal experimental protocols were approved by the Nanjing University of Chinese Medicine Committee on Laboratory Animal Care and all animals received humane care according to the National Institutes of Health guidelines. The animals were housed under diurnal lighting conditions (12:12) and had access to food and water ad libitum. Female Sprague-Dawley rats (weighting 200 \pm 30 g) were provided by Zhejiang Experimental Animal Center (Nanjing, China).

Cell proliferation assay. Cell proliferation was analyzed using the MTT assay. VSMCs $\left(1 \times 10^{4} /\right.$ well) were seeded in a 96-well microplate and were cultured with $200 \mu 1$ DMEM supplemented with $10 \%$ FBS. Once the cells had reached $60 \%$ confluence they were serum-starved for $16 \mathrm{~h}$ at $37^{\circ} \mathrm{C}$ in a humidified chamber containing $5 \% \mathrm{CO}_{2}$. VSMCs were then treated with $100 \mathrm{nM}$ Ang II or $100 \mathrm{nM}$ Ang II + LWDHF (3, 6 and $12 \mu \mathrm{g} / \mathrm{ml}$ ) for $24 \mathrm{~h}$; cells were incubated with MTT $(5 \mathrm{mg} /$ $\mathrm{ml}$ ) for the last $4 \mathrm{~h}$ at $37^{\circ} \mathrm{C}$ in a humidified chamber containing $5 \% \mathrm{CO}_{2}$ and then dissolved into $150 \mu \mathrm{l}$ DMSO. Untreated 
cells served as the control group. MTT was dissolved in PBS at a concentration of $5 \mathrm{mg} / \mathrm{ml}$. Subsequently, optical density was measured at $490 \mathrm{~nm}$ using a Universal Microplate Spectrophotometer (BioTek Instruments, Inc., Winooski, VT, USA). All experiments were performed in triplicate.

Western blot analysis. Cells were lysed in radioimmunoprecipitation assay (RIPA) buffer with a protease and phosphatase inhibitor cocktail (protease inhibitor : phosphatase inhibitor $=100: 1 / 1)$. Solubilized protein samples were centrifuged at $12,000 \mathrm{x} \mathrm{g}$ for $15 \mathrm{~min}$ at $4^{\circ} \mathrm{C}$, and protein concentration within the supernatant was determined using a Bradford protein assay. Equal amounts of protein (30-50 $\mu \mathrm{g})$ were separated by $10 \%$ SDS-PAGE and were transferred to polyvinylidene fluoride membranes (EMD Millipore, Billerica, MA, USA). The membranes were then blocked with $5 \%$ non-fat milk powder in Tris-buffered saline containing $0.1 \%$ Tween-20 (TBST) for $2 \mathrm{~h}$ at room temperature, and were incubated with various primary antibodies (all antibodies were diluted in 1X TBST) overnight at $4^{\circ} \mathrm{C}$. After washing with TBST, the membranes were incubated for $90 \mathrm{~min}$ at room temperature with a rabbit anti-goat secondary antibody (sc-2004; 1:5,000; Santa Cruz Biotechnology, Inc.). After washing three times with TBST, the membranes were detected using a chemiluminescence kit (EMD Millipore). The blots were finally semi-quantified by densitometric analysis (Image Lab Software, version 3.0 Beta 3). Specific protein expression levels were normalized to $\beta$-tubulin or $\beta$-actin for total protein analyses.

Reverse transcription-quantitative polymerase chain reaction (RT-qPCR). Total RNA from VSMCs was isolated using TRIzol reagent (Invitrogen; Thermo Fisher Scientific, Inc., Waltham, MA, USA). RNA concentration was quantified by measuring the absorbance at $260 \mathrm{~nm}$ using a spectrophotometer, and RNA purity was assessed according to the 260 and $280 \mathrm{~nm}$ ratio. RT was conducted according to the manufacturer's protocol. RNA $(5 \mu \mathrm{g})$ was then reverse transcribed into cDNA using a First Strand cDNA Synthesis kit (Thermo Fisher Scientific, Inc.). The obtained cDNA was used to determine the mRNA expression levels of $\alpha$-SMA, OPN, ER $\alpha$ and ER $\beta$ by qPCR using a Thermo Fisher Scientific ABI7500 instrument (Thermo Fisher Scientific, Inc.) with SuperScript II First-Strand Synthesis SuperMix (Thermo Fisher Scientific, Inc.), and relative target gene expression was normalized to GAPDH or $\beta$-tubulin. The primer sequences were designed by Biogot Technology Co., Ltd. (Nanjing, China) and are presented in Table I. RT-qPCR was performed using AceQ qPCR SYBR Green Master Mix. Amplification was initiated at $95^{\circ} \mathrm{C}$ for $10 \mathrm{~min}$, followed by 40 cycles at $95^{\circ} \mathrm{C}$ for $10 \mathrm{sec}$, and was finally terminated at $60^{\circ} \mathrm{C}$ for $40 \mathrm{sec}$ and analysed using the $\Delta \Delta \mathrm{Ct}$ method on an ABI 7500 Thermal Cycler (both from Invitrogen; Thermo Fisher Scientific, Inc.). The quantification cycle $(\mathrm{Cq})$ value of the gene of interest was obtained and was initially normalized to the housekeeping gene GAPDH or $\beta$-tubulin $(\Delta \mathrm{Cq})$, after which the value was further normalized to the control $(\Delta \Delta \mathrm{Cq})$. The fold-change of each target gene was calculated using the $2^{-\Delta \Delta \mathrm{Cq}}$ method (24).

Transmission electron microscopy. The cells were fixed in $2 \%$ glutaraldehyde in neutral phosphate buffer, post-fixed in osmium tetraoxide, and embedded in Epon. Sections were cut at $80 \mathrm{~nm}$ and examined under a Philips Tecnai 10 electron microscope.

Flow cytometry. Primary VSMCs were seeded in 6-well plates at a density of $2 \times 10^{5}$ cells and were incubated at $37^{\circ} \mathrm{C}$ for $24 \mathrm{~h}$. After VSMCs were treated with Ang II and/or LWDHF at $37^{\circ} \mathrm{C}$ for $24 \mathrm{~h}$, they were trypsinized, collected and washed twice with cold PBS at $4^{\circ} \mathrm{C}$ for $5 \mathrm{~min}$. Precipitated cells were fixed in $1 \mathrm{ml}$ ice-cold $70 \%$ ethanol overnight at $4^{\circ} \mathrm{C}$. The fixed cells were then washed in PBS, treated with RNase A $(10 \mu \mathrm{g} /$ $\mathrm{ml})$, and DNA was stained with propidium iodide $(50 \mu \mathrm{g} /$ $\mathrm{ml})$ for $30 \mathrm{~min}$ at $37^{\circ} \mathrm{C}$ in the dark. Cells $\left(1 \times 10^{4}\right)$ were finally analyzed by flow cytometry (FACSCalibur; BD Biosciences, San Jose, CA, USA)

Wound-healing assay. Primary VSMCs were seeded in 6-well plates at a density of $2 \times 10^{5}$ cells for $24 \mathrm{~h}$ to ensure a single cell distribution. Confluent cells were scratched along the edge of the attached cells to create a double-sided wound using a $10 \mu 1$ plastic pipette tip and were rinsed twice with PBS to remove cell debris. Cells were then grown in serum-free medium supplemented with Ang II and/or LWDHF for $24 \mathrm{~h}$. Cell gaps were observed under a Zeiss microscope (CarlZeiss, Hallbergnoos, Germany) and images were captured of the wound sites. VSMC migration was assessed on the basis of the injured area covered by cells counted from the wound borders.

Transwell migration assay. The effects of LWDHF on VSMC migration were examined by Transwell chamber assay. Primary VSMCs cells $\left(1 \times 10^{5}\right.$ cells/well $)$ at passages 3-7 were seeded in a $36 \mathrm{~mm}$ culture dish for $24 \mathrm{~h}$. Cells were then trypsinized, resuspended in $0.5 \%$ FBS medium in the upper chamber and incubated with LWDHF $(12 \mu \mathrm{g} / \mathrm{ml})$, DMEM medium containing Ang II (100 nM) was added to the lower chamber. The cells were incubated at $37^{\circ} \mathrm{C}$ in an atmosphere containing $5 \% \mathrm{CO}_{2}$ for $24 \mathrm{~h}$ and were allowed to migrate through the micropores to the bottom side of the Transwell apparatus. The remaining cells in the upper chamber were removed with a cotton swab, the cell membrane surface was wiped, and the lower side of the filter harboring the migrated VSMCs was fixed with $4 \%$ paraformaldehyde for $30 \mathrm{~min}$. The migrating cells were then stained with $0.5 \%$ Coomassie Brilliant Blue for $10 \mathrm{~min}$. Stained cells in five random visual fields from each of the Transwell filters were selected and images were captured under a Zeiss microscope (x200 magnification; CarlZeiss).

Analysis of F-actin cytoskeleton by fluorescence microscopy. Primary VSMCs were cultured on glass coverslips treated with indicated agents at $37^{\circ} \mathrm{C}$ for $24 \mathrm{~h}$. Cells were then fixed with pre-cooled $4 \%$ paraformaldehyde and rinsed three times with PBS at room temperature. Cells were permeabilized in $0.1 \%$ Triton $X-100$ and incubated with $1 \%$ bovine serum albumin (10735108001; Biosharp, Hefei, China)/PBS to block nonspecific binding, after which they were incubated with phalloidin-fluorescein isothiocyanate for $60 \mathrm{~min}$ at room temperature in the dark. After three washes with PBS at $4^{\circ} \mathrm{C}$ for 5 min, images were captured and were analyzed using the ZEN 2011 imaging software on a Zeiss inverted microscope (Carl Zeiss AG, Oberkochen, Germany) under 400-fold magnification. 
Table I. Primer sequences used for polymerase chain reaction.

\begin{tabular}{lll}
\hline Gene & \multicolumn{1}{c}{ Sense primer } & \multicolumn{1}{c}{ Antisense primer } \\
\hline$\beta$-tubulin & 5-CGCAAGCTAGCTGTCAACAT-3 & 5-CTGCTCATCCACCTCCTTCA-3 \\
$\alpha$-SMA & 5-CATCATGCGTCTGGACTTGG-3 & 5-CCAGGGAAGAAGAGGAAGCA-3 \\
OPN & 5-AGCCATGAGTCAAGTCAGCT-3 & 5-ACTCGCCTGACTGTCGATAG-3 \\
GAPDH & 5-CCTCTATGCCAACACAGTGC-3 & 5-CCTGCTTGCTGATCCACATC-3 \\
ER $\alpha$ & 5-TCGCTACTGTGCTGTGTGTA-3 & 5-GCCTTTCATCATGCCCACTT-3 \\
ER $\beta$ & 5-TGAGCACCTTGAGTCCAGAG-3 & 5-AGTCCCACCATTAGCACCTC-3
\end{tabular}

$\alpha$-SMA, $\alpha$-smooth muscle actin ; ER, estrogen receptor; OPN, osteopontin.

Transfection of SiRNA. Human VSMCs were seeded in a 6 -well culture plate at a density of $2 \times 10^{5}$ cells in $2 \mathrm{ml}$ antibiotic-free normal growth medium supplemented with $10 \% \mathrm{FBS}$. The cells were incubated at $37^{\circ} \mathrm{C}$ in a $\mathrm{CO}_{2}$ incubator until the cells reached $60 \%$ confluence. Cells were then transfected with a transfection mixture composed of ER $\alpha$ siRNA (sc-29305) or control siRNA (sc-37007) and siRNA transfection reagent (sc-29528) (all Santa Cruz Biotechnology, Inc.) according to the manufacturer's protocols. After $6 \mathrm{~h}$, cells were washed and cultured for $18 \mathrm{~h}$ in complete medium, and were treated with Ang II and/or LWDHF for $24 \mathrm{~h}$. The cells were subsequently lysed and collected for western blot analyses.

Statistical analysis. SPSS 10.0 (SPSS, Inc., Chicago, IL, USA) was used for all statistical analyses. All data are presented as the mean \pm standard deviation of at least three independent experiments. Differences in the results between two groups were evaluated using either two-tailed Student's t-test or one-way ANOVA followed by Dunnett's post hoc test. $\mathrm{P}<0.05$ was considered to indicate a statistically significant difference.

\section{Results}

LWDHF inhibits Ang II-induced VSMC proliferation and induces cell cycle arrest. The present study evaluated the effects of LWDHF on normal primary VSMC proliferation using the MTT assay. Growth-arrested cells were treated with LWDHF (0.1-100 g/l) in the presence of $10 \% \mathrm{FBS}$; the results indicated that no significant difference in cell viability was observed following treatment with $\leq 10 \mathrm{~g} / 1 \mathrm{LWDHF}$ (Fig. 2A), suggesting that $\leq 10 \mathrm{~g} / 1 \mathrm{LWDHF}$ did not exert significant cytotoxicity to normal cells. However, treatment with Ang II (100 nM) induced a 1.45-fold increase in VSMC proliferation, whereas treatment with LWDHF attenuated the effects of Ang II on VSMC proliferation in a dose-dependent manner (Fig. 2B); treatment with the highest concentration of LWDHF $(12 \mu \mathrm{g} / \mathrm{ml})$ significantly inhibited Ang II-induced VSMC proliferation. In addition, flow cytometric analysis was performed to observe the effects of LWDHF on cycle progression of VSMCs. As shown in Table II, the percentage of cells at $\mathrm{G}_{0} / \mathrm{G}_{1}, \mathrm{~S}$ and $\mathrm{G}_{2} / \mathrm{M}$ phases in the Ang II-stimulated group was $58.85 \pm 5.01,21.82 \pm 2.19$ and $19.34 \pm 2.86 \%$, respectively. LWDHF, at concentrations of 3,6 and $12 \mu \mathrm{g} / \mathrm{ml}$, effectively increased the proportion of cells in the $G_{0} / G_{1}$ phase and simul-
Table II. Effects of LWDHF on Ang II-induced cell cycle progression.

\begin{tabular}{lcccc}
\hline & & \multicolumn{4}{c}{ Phases } \\
\cline { 3 - 5 } Group & Dose & $\mathrm{G}_{0} / \mathrm{G}_{1}$ & $\mathrm{~S}$ & $\mathrm{G}_{2} / \mathrm{M}$ \\
\hline Control & & $70.81 \pm 0.39^{\mathrm{a}}$ & $16.24 \pm 0.53^{\mathrm{a}}$ & $12.96 \pm 0.44^{\mathrm{a}}$ \\
Ang II & $100 \mathrm{nM}$ & $58.85 \pm 5.01$ & $21.82 \pm 2.19$ & $19.34 \pm 2.86$ \\
LWDHF & $3 \mu \mathrm{g} / \mathrm{ml}$ & $67.01 \pm 0.27^{\mathrm{b}}$ & $19.20 \pm 1.03$ & $13.79 \pm 0.77^{\mathrm{a}}$ \\
& $6 \mu \mathrm{g} / \mathrm{ml}$ & $67.90 \pm 0.47^{\mathrm{b}}$ & $18.27 \pm 0.59^{\mathrm{b}}$ & $13.84 \pm 0.37^{\mathrm{a}}$ \\
& $12 \mu \mathrm{g} / \mathrm{ml}$ & $68.73 \pm 0.29^{\mathrm{a}}$ & $17.68 \pm 0.42^{\mathrm{b}}$ & $13.59 \pm 0.61^{\mathrm{a}}$ \\
\hline
\end{tabular}

Data are presented as the mean \pm standard deviation, $n=4$. ${ }^{\mathrm{a}} \mathrm{P}<0.01$, ${ }^{\mathrm{b}} \mathrm{P}<0.05$ vs. the Ang II-stimulated group. Ang II, angiotensin II; LWDHF, Liuwei Dihuang formula.

taneously decreased the $\mathrm{S}$ and $\mathrm{G}_{2} / \mathrm{M}$ phase cell populations. These results indicated that LWDHF may exert suppressive effects on Ang II-induced VSMC proliferation; these effects may be associated with the induction of cell cycle arrest at $\mathrm{G}_{0} / \mathrm{G}_{1}$ phase.

LWDHF suppresses Ang II-stimulated VSMC migration. The effects of LWDHF on Ang II-stimulated VSMC migration were observed using a wound-healing assay. Equal numbers of confluent VSMCs were scratched to create a double-sided wound using a $10 \mu$ l pipette tip. The cells were then treated with Ang II (100 nM) or Ang II $(100 \mathrm{nM})+\operatorname{LWDHF}(12 \mu \mathrm{g} / \mathrm{ml})$. As presented in Fig. 3A, Ang II promoted VSMCs to migrate into the wound, whereas cell migration was reduced in the LWDHF-treated group compared with in the Ang II-stimulated group, thus suggesting that LWDHF may reduce VSMC migration. In addition, a Transwell chamber assay was conducted to verify the effects of LWDHF on Ang II-induced VSMC migration. As shown in Fig. 3B, LWDHF treatment markedly decreased migration of VSMCs to the bottom chamber in response to Ang II compared with those treated with Ang II alone. Taken together, these results indicated that LWDHF may reduce Ang II-induced VSMC migration.

Effects of LWDHF on Ang II-induced F-actin organization in VSMCs. The present study demonstrated that LWDHF 


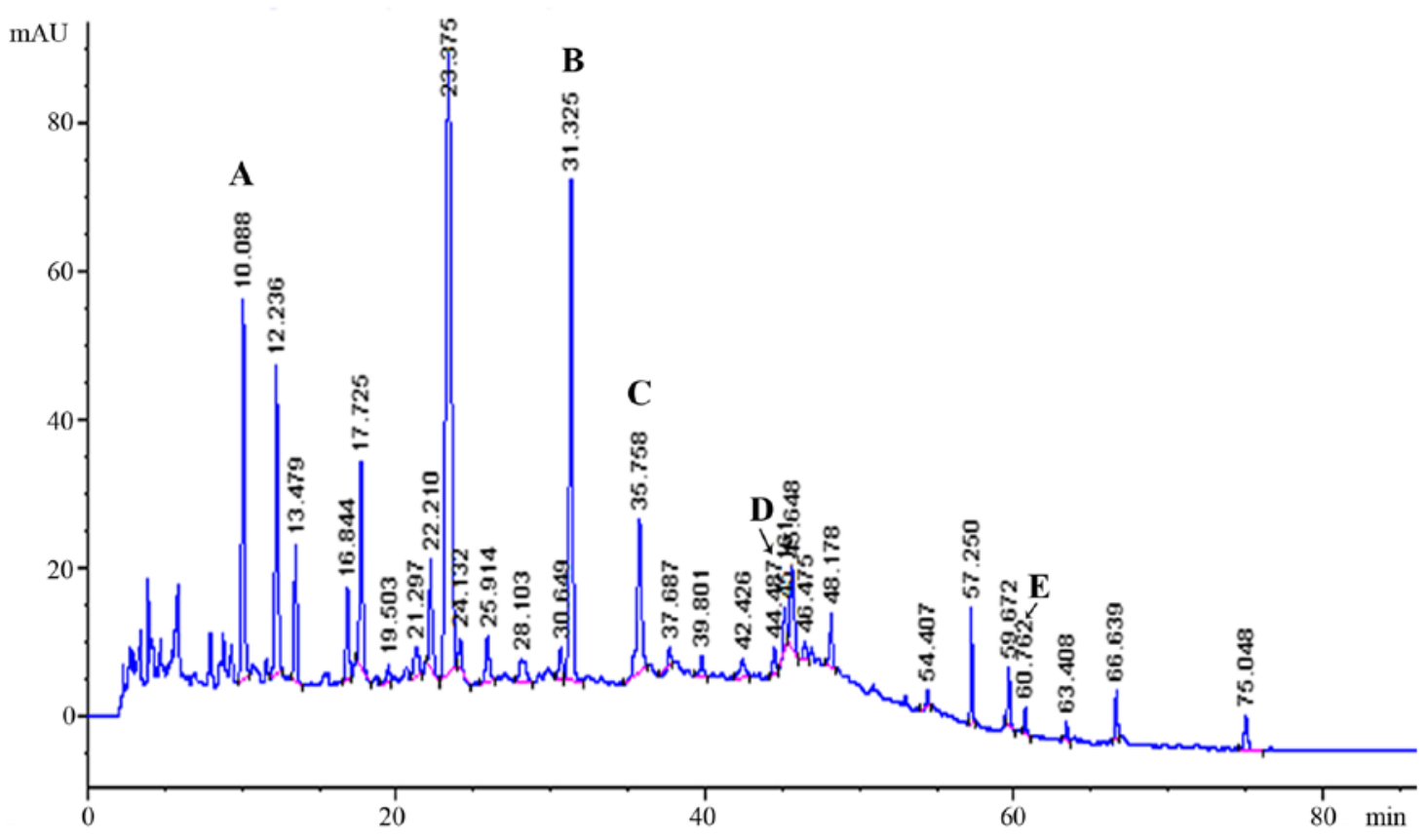

Figure 1. Representative high-performance liquid chromatograms of Liuwei Dihuang formula. (A) Gallic acid; (B) loganin; (C) paeoniflorin; (D) verbascoside; and (E) paeonoside.

A

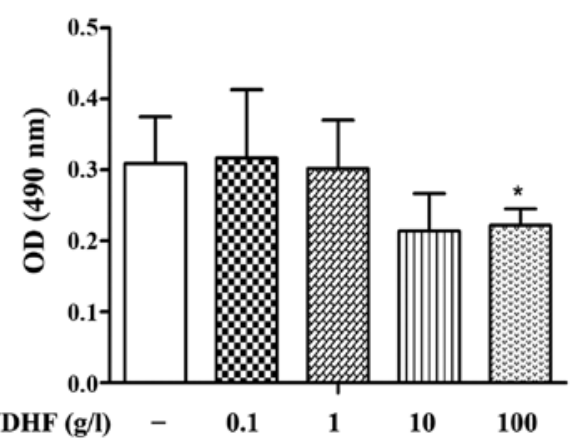

B

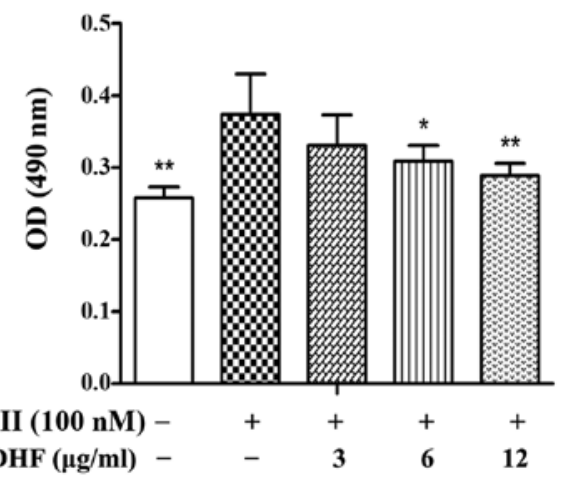

Figure 2. Effects of LWDHF on Ang II-induced VSMC proliferation. (A) MTT assays were performed to determine the effects of LWDHF on the proliferation of normal VSMCs stimulated by $10 \%$ fetal bovine serum. Relative proliferation rate was displayed using control cells as a standard $(\mathrm{n}=6)$. ${ }^{*} \mathrm{P}<0.05 \mathrm{vs}$. the control group. (B) MTT assays were performed to determine the rate of VSMC proliferation in each group. Relative proliferation rate was displayed using Ang II-stimulated cells as a standard $(\mathrm{n}=6) .{ }^{*} \mathrm{P}<0.05,{ }^{* * *} \mathrm{P}<0.01$ vs. the Ang II-stimulated group. Ang II, angiotensin II; LWDHF, Liuwei Dihuang formula; OD, optical density; VSMC, vascular smooth muscle cell.

inhibited the migration of Ang II-stimulated VSMCs. Therefore, in order to determine whether LWDHF decreased cell migration by influencing reorganization of the cytoskeleton, F-actin was visualized by phalloidin staining, $24 \mathrm{~h}$ after Ang II (100 nM)-stimulated VSMC spreading. Treatment with Ang II resulted in a substantial increase in the number of stress fibers, as well as the rearrangement of these structures into ordered parallel arrays, in cultured VSMCs. Conversely, treatment with LWDHF $(12 \mu \mathrm{g} / \mathrm{ml})$ abolished reorganization of the actin cytoskeleton induced by Ang II (Fig. 3C).

LWDHF inhibits Ang II-induced VSMC phenotypic switching. VSMCs transform from a differentiated contractile phenotype into a synthetic phenotype, which is associated with low expression of contractile proteins and high expression of rough endoplasmic reticulum (RER) and ECM proteins (25). The ultrastructure of VSMCs was observed under transmission electron microscopy (Fig. 4). The cytoplasm in normal VSMCs was filled with myofilaments, while homogeneous distribution of chromatin in nucleus. In cells treated with Ang II for $24 \mathrm{~h}$, the the muscle filament in the cytoplasm obviously decreased compared to that in the control group, but the Golgi bodyobviously increased, and the endoplasmic reticulum was obviously dilated. These ultrastructural alterations are characteristics of the synthetic phenotype, thus suggesting that Ang II induced phenotypic modulation of VSMCs. However, after treatment with LWDHF ( $12 \mu \mathrm{g} / \mathrm{ml})$, the myocytes in the cytoplasm were still rich, but the organelles of the Golgi bodies were decreased. These ultrastructural observations indicated that LWDHF reversed Ang II-induced VSMC phenotypic switching. Furthermore, the present study measured alterations in the expression of molecular markers associated with contractile 

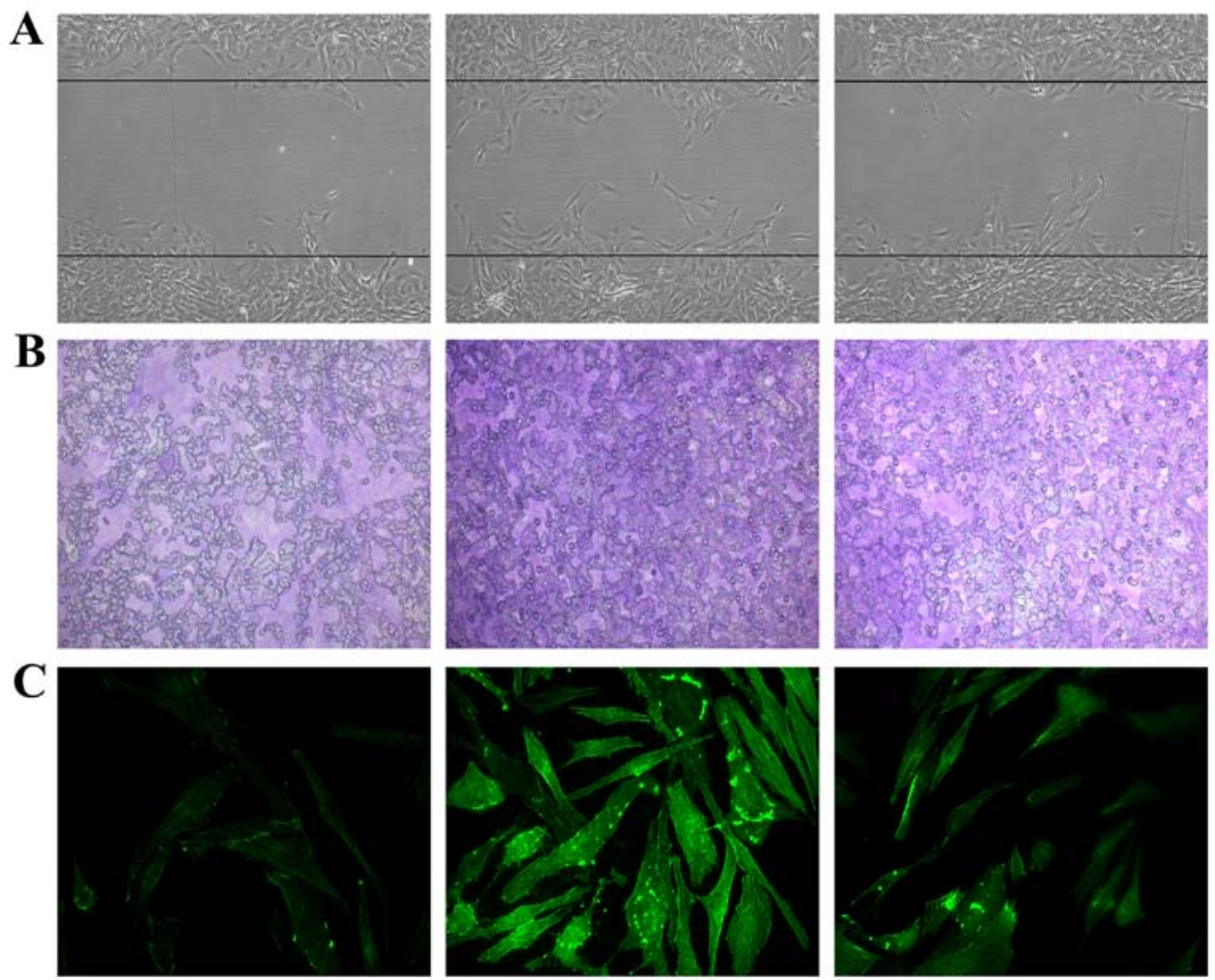

Control

Ang II (100 nM)

LWDHF (12 $\mu \mathrm{g} / \mathrm{ml})$

Figure 3. Effects of LWDHF (12 $\mu \mathrm{g} / \mathrm{ml})$ on VSMC migration in response to Ang II (100 nM). (A) Cells were cultured in the presence of $10 \%$ FBS. After generation of a single scratch, medium was replaced with medium containing $2 \%$ FBS and cells were treated with Ang II with or without LWDHF. Cell wounds were observed under a microscope (x40 magnification). (B) Primary VSMCs were seeded in the upper chamber of a modified Boyden chamber and were treated with Ang II (100 nM) with or without LWDHF for $24 \mathrm{~h}$. Untreated cells were used as a control. Images of five random visual fields from the bottom chamber of each group were captured under a microscope (x200 magnification). (C) Inhibitory effects of LWDHF (12 $\mu \mathrm{g} / \mathrm{ml})$ on reorganization of the actin cytoskeleton following treatment with Ang II (100 nM) for $24 \mathrm{~h}$. The cells were fixed, stained with fluorescein isothiocyanate-labeled phalloidin and were examined by fluorescence microscopy. Untreated cells were used as a control. Images of five random visual fields from each group were captured under a microscope (x200 magnification). Ang II, angiotensin II; FBS, fetal bovine serum; LWDHF, Liuwei Dihuang formula; VSMCs, vascular smooth muscle cells.

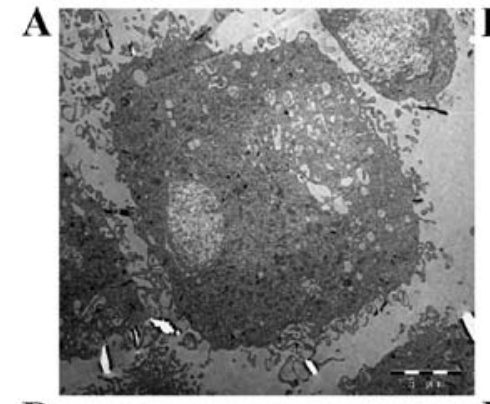

D

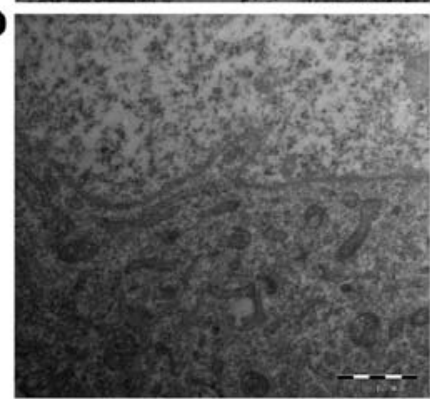

Control
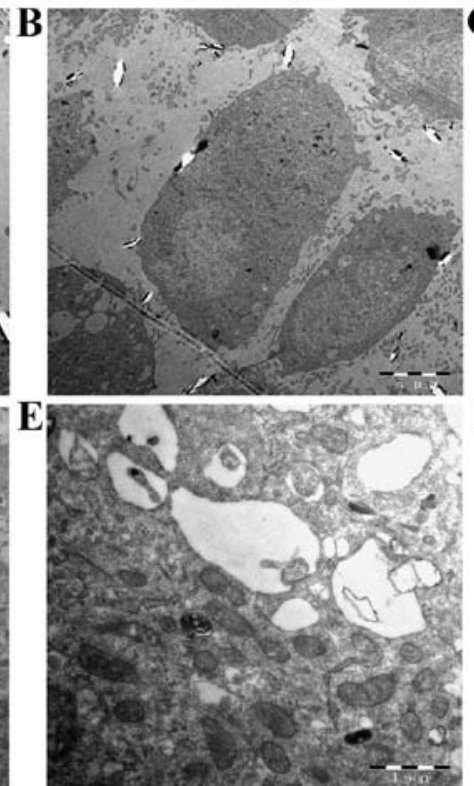

Ang II (100 nM)
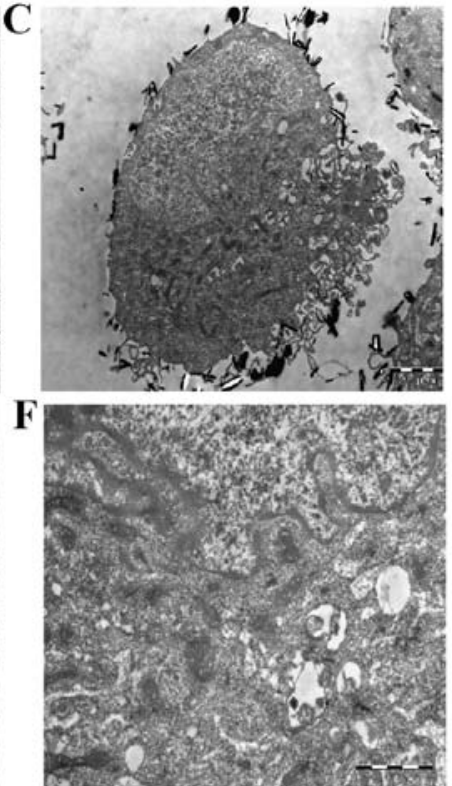

LWDHF $(12 \mu \mathrm{g} / \mathrm{ml})$

Figure 4. Ultrastructural alterations of VSMCs observed by transmission electron microscopy. (A and D) Representative images of the cytoplasm from normal VSMCs; (B and E) VSMCs treated with Ang II $(100 \mathrm{nM})$ for $24 \mathrm{~h}$. The numbers of rough endoplasmic reticulum and Golgi apparatus were increased in the cytoplasm, and the nuclei were larger compared with in untreated cells. (C and F) Following treatment with LWDHF, the nuclear shape became regular, and the numbers of rough endoplasmic reticulum and Golgi apparatus were decreased in the cytoplasm (A-C) x5,000 magnification; (D-F) x30,000 magnification. Ang II, angiotensin II; LWDHF, Liuwei Dihuang formula; VSMCs, vascular smooth muscle cells. 
$\mathbf{A}$

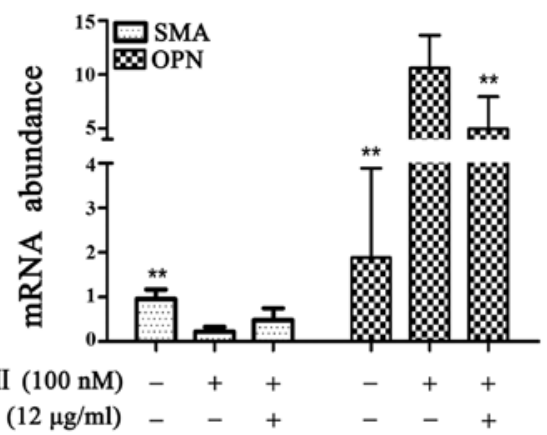

B

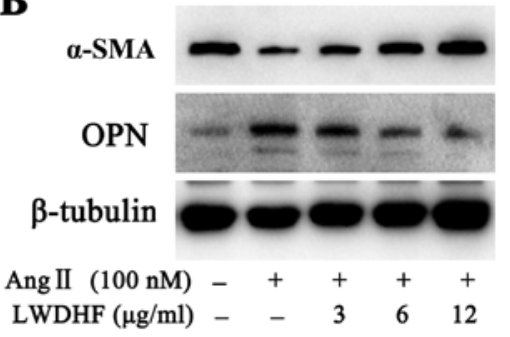

$\mathbf{C}$

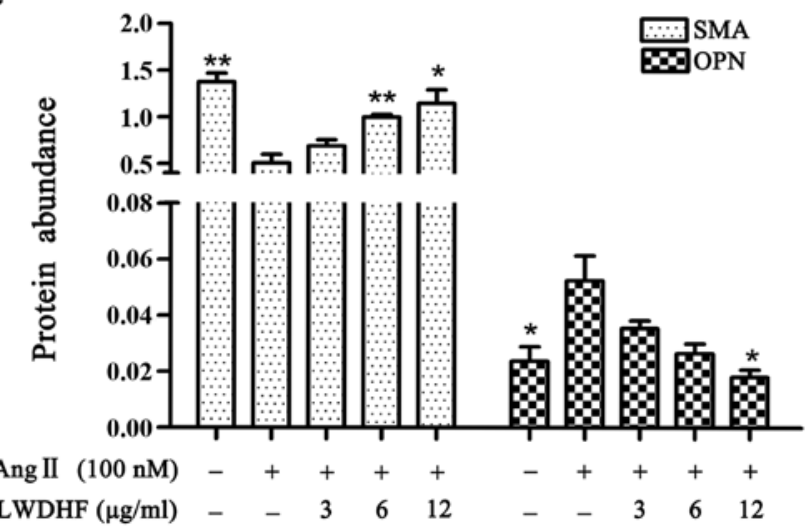

Figure 5. Effects of LWDHF on Ang II-induced contractile-to-synthetic phenotypic switching. (A) mRNA expression levels of $\alpha$-SMA and OPN were measured by reverse transcription-quantitative polymerase chain reaction. GAPDH was used as an internal control ( $\mathrm{n}=3$ ). (B) Western blot analysis was performed to determine the expression levels of contractile and synthetic proteins. $\beta$-tubulin was used as an internal control (n=3). (C) Histograms represent relative protein expression levels. Data are presented as the mean \pm standard error of the mean. ${ }^{*} \mathrm{P}<0.01,{ }^{* *} \mathrm{P}<0.05$ vs. Ang II (100 nM)-stimulated group. $\alpha-\mathrm{SMA}$, $\alpha$-smooth muscle actin; Ang II, angiotensin II; LWDHF, Liuwei Dihuang formula; OPN, osteopontin; VSMC, vascular smooth muscle cell.

and synthetic VSMC phenotypes. RT-qPCR and western blot analysis were used to measure the relative mRNA and protein abundance of $\alpha$-SMA and OPN, respectively. The results indicated that exposure of VSMCs to Ang II for $24 \mathrm{~h}$ resulted in a $75 \%$ decrease in the mRNA expression levels of $\alpha$-SMA and a robust upregulation of OPN (5-fold) (Fig. 5A). In addition, a decline in the protein expression levels of $\alpha$-SMA and an increase in OPN protein expression were determined by western blotting (Fig. 5B and C). Conversely, addition of LWDHF dose-dependently rescued the downregulation of $\alpha$-SMA and abolished the upregulation of OPN in Ang II-treated VSMCs. These results confirmed that the aforementioned ultrastructural alterations were due to LWDHF-induced inhibition of VSMC phenotypic transition caused by Ang II, and indicated that LWDHF suppressed VSMC proliferation and migration via inhibiting VSMC phenotypic switching.

LWDHF regulates expression of ERs in VSMCs. Our previous studies demonstrated that LWDHF exerts estrogen-like effects on the prevention of cardiovascular diseases in experimental rat models $(15,16)$. The present study investigated whether LWDHF could affect the expression of ERs in VSMCs. As shown in Fig. 6, the mRNA expression levels of ER $\alpha$ and ER $\beta$ were significantly downregulated in VSMCs stimulated with Ang II (100 nM) compared with in untreated cells. Conversely, VSMCs treated with LWDHF and exposed to Ang II for $24 \mathrm{~h}$ exhibited a significant upregulation of $\mathrm{ER} \alpha$; in addition ER $\beta$ exhibited higher expression in LWDHF-treated cells compared with in cells treated with Ang II alone. The results of western blotting were consistent with those of RT-qPCR.

Role of ERs in Ang II-induced phenotypic modulation of VSMCs. To examine whether ERs were involved in the inhibitory effects of LWDHF on the phenotypic modulation of VSMCs, tamoxifen, a nonselective estrogen antagonist, was used to characterize the role of ER in LWDHF-mediated effects. As shown in Fig. 7A, part suppression of ER signaling by tamoxifen markedly attenuated the inhibitory effects of LWDHF on VSMC phenotypic switching; the ability of LWDHF to increase $\alpha$-SMA protein expression and decrease OPN protein expression was significantly abrogated by tamoxifen. Furthermore, human aortic VSMCs (HAVSMCs) were transfected with ER $\alpha$ siRNA to observe the role of $\mathrm{ER} \alpha$ in LWDHF-mediated inhibition of VSMC phenotypic switching. When $0.24 \mathrm{nM}$ ER $\alpha$ siRNA was transfected into HAVSMCs, the constitutive expression of ER $\alpha$ was significantly reduced after $6 \mathrm{~h}$ (Fig. 7B), indicating that HAVSMCs were successfully transfected with this siRNA. HAVSMCs were transfected with ER $\alpha$ siRNA for $6 \mathrm{~h}$ and were then stimulated with Ang II (100 nM) for $24 \mathrm{~h}$; these cells exhibited a significant reduction in $\alpha$-SMA expression and a significant increase in OPN expression compared with in cells transfected with control siRNA and treated with Ang II. In addition, HAVSMCs transfected with ER $\alpha$ siRNA and stimulated with Ang II in the presence of LWDHF for 24 h exhibited weakened upregulated SMA expression, while 
A
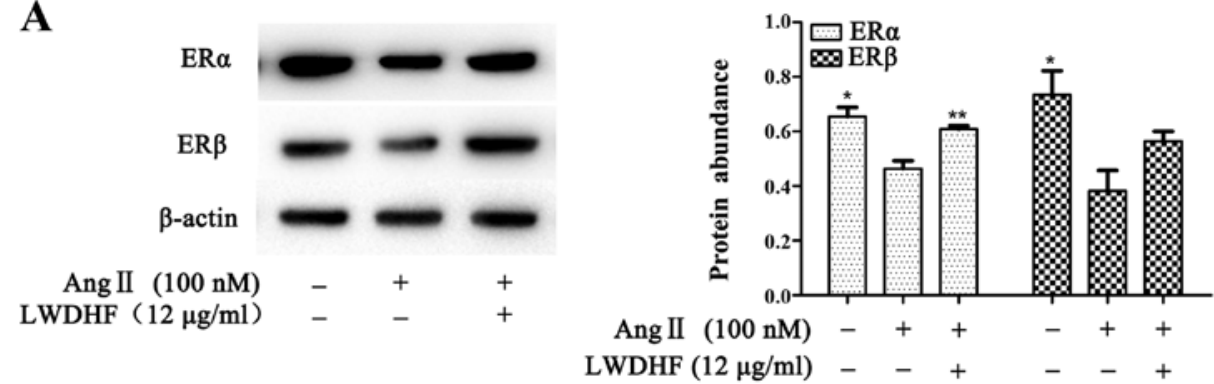

B

LWDHF $(12 \mu \mathrm{g} / \mathrm{ml})-{ }_{-}+-+$

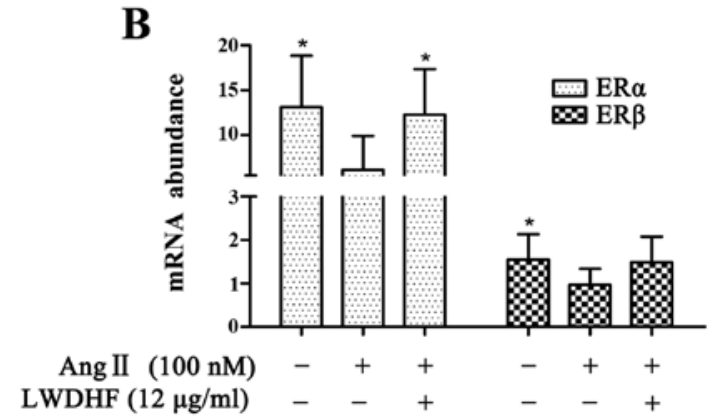

Figure 6. LWDHF suppresses Ang II-induced downregulation of ER expression. (A) VSMCs were treated with normal saline or Ang II (100 nM) in the absence or presence of LWDHF (12 $\mu \mathrm{g} / \mathrm{ml})$. ER $\alpha$ and ER $\beta$ expression was detected by western blot analysis (left panel). ER $\alpha$ and ER $\beta$ protein expression was normalized to $\beta$-actin (right panel) ( $\mathrm{n}=3$ ). Data are presented as the mean \pm standard error of the mean. ${ }^{*} \mathrm{P}<0.05,{ }^{* *} \mathrm{P}<0.01$ vs. Ang II-stimulated group. (B) mRNA expression levels of ER $\alpha$ and ER $\beta$ were measured by reverse transcription-quantitative polymerase chain reaction. $\beta$-actin was used as an internal control $(\mathrm{n}=3)$. Data are presented as the mean \pm standard deviation. ${ }^{*} \mathrm{P}<0.05$ vs. Ang II-stimulated group. Ang II, angiotensin II; ER, estrogen receptor; Liuwei Dihuang formula; VSMC, vascular smooth muscle cell.

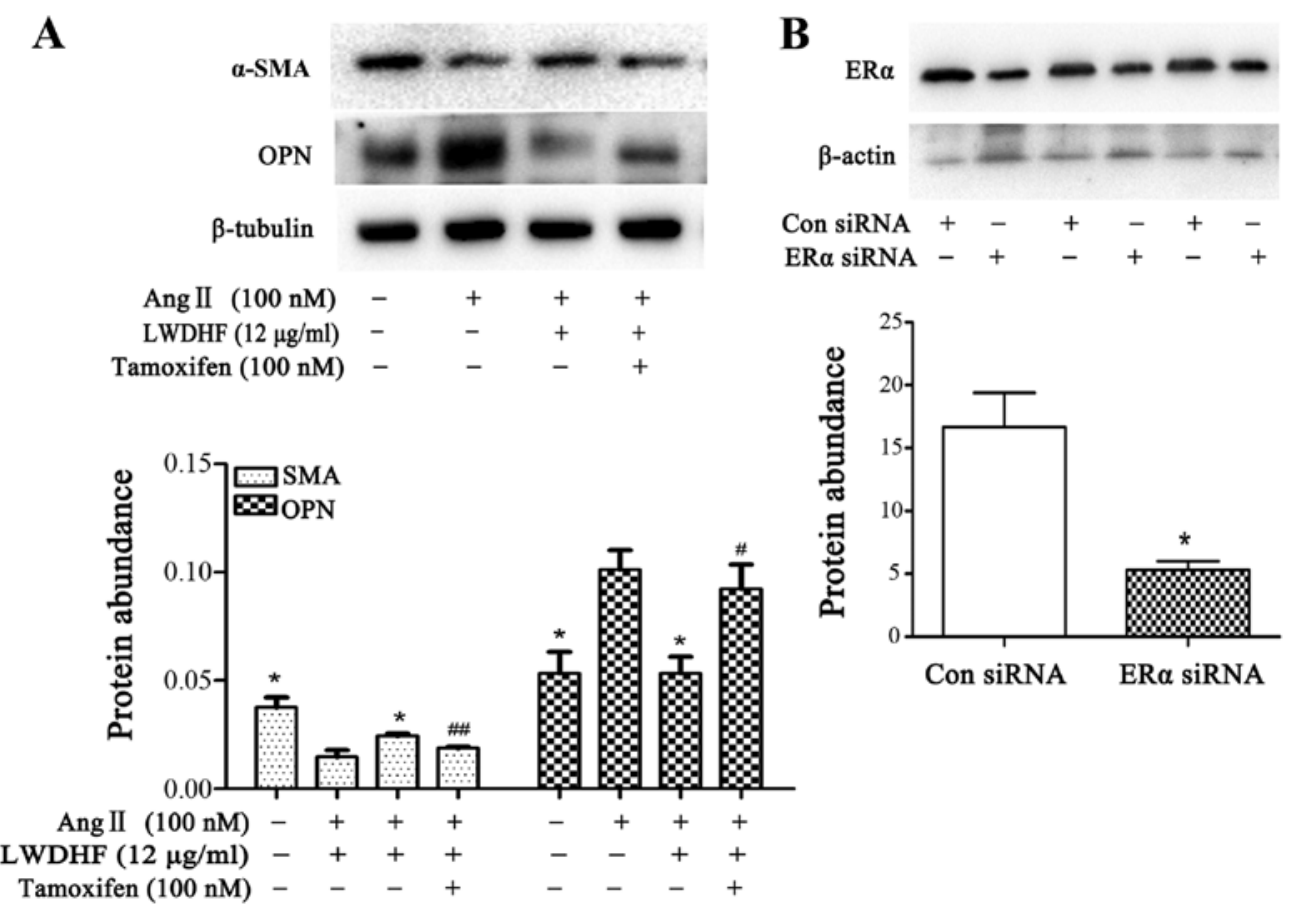

Figure 7. ER $\alpha$ knockdown significantly attenuates the effects of LWDHF on phenotypic switching of VSMCs. (A) LWDHF-induced inhibition of VSMC phenotypic switching was reversed by tamoxifen. Cells were treated with or without Ang II at $100 \mathrm{nM}$ in the absence or presence of LWDHF $(12 \mu \mathrm{g} / \mathrm{ml})$ or tamoxifen $(100 \mathrm{nM})(\mathrm{n}=3)$. Data are presented as the mean \pm standard error of the mean. ${ }^{*} \mathrm{P}<0.05$ vs. Ang II-stimulated group; ${ }^{\#} \mathrm{P}<0.05$, ${ }^{\# \#} \mathrm{P}<0.01$ vs. Ang II + LWDHF-treated group. (B) Cells were transfected with ER $\alpha$ siRNA to silence ER $\alpha$. ER $\alpha$ expression was significantly decreased (n=3). Data are presented as the mean \pm standard error of the mean. " $\mathrm{P}<0.05$ vs. control siRNA-transfected cells. Ang II, angiotensin II; ER, estrogen receptor; Liuwei Dihuang formula; siRNA, small interfering RNA; VSMC, vascular smooth muscle cell.

the expression of OPN was not downregulated. The results showed that the expression of SMA and OPN was related to ER $\alpha$, and the change of SMA and OPN expression were mediated by ER $\alpha$ (Fig. 8). Taken together, these results indicated that ER $\alpha$ knockdown abolished the inhibitory effects of LWDHF on Ang II-induced VSMC phenotypic switching, 
A

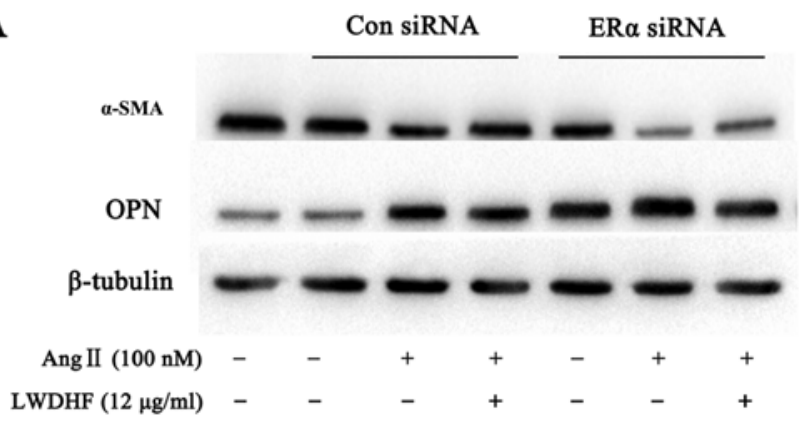

B

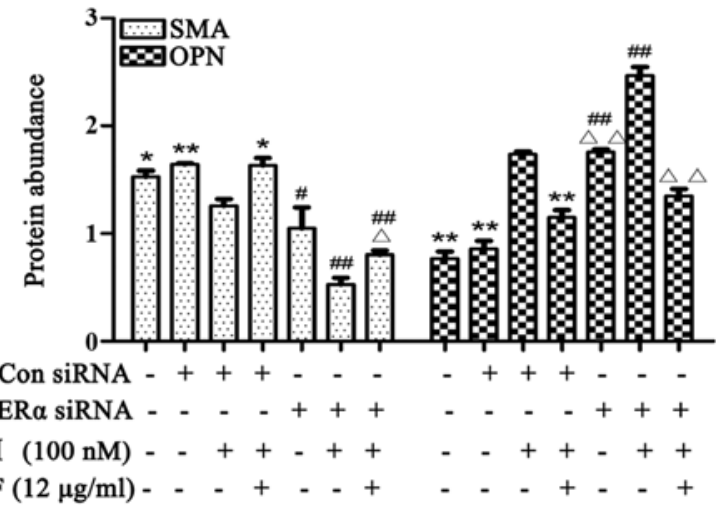

Figure 8. ER $\alpha$ knockdown significantly attenuates the effects of LWDHF on phenotypic modulation of vascular smooth muscle cells. (A) After $6 \mathrm{~h}$ transfection, cells were treated with $100 \mathrm{nM}$ Ang II in the absence or presence of LWDHF $(12 \mu \mathrm{g} / \mathrm{ml})$. Western blot analysis was performed to determine the protein expression levels of $\alpha$-SMA and OPN. (B) Relative protein expression levels of $\alpha$-SMA and OPN were normalized relative to $\beta$-tubulin levels. Data are presented as the mean \pm standard error of the mean $(n=3)$. ${ }^{*} \mathrm{P}<0.05$, ${ }^{* *} \mathrm{P}<0.01$ vs. control siRNA + Ang II group; ${ }^{\#} \mathrm{P}<0.05$, ${ }^{\# \#} \mathrm{P}<0.01$ vs. control siRNA group; ${ }^{\Delta} \mathrm{P}<0.05,{ }^{\Delta \Delta} \mathrm{P}<0.01$ vs. ER $\alpha$ siRNA + Ang II group. $\alpha$-SMA $\alpha$-smooth muscle actin; Ang II, angiotensin II; ER, estrogen receptor Liuwei Dihuang formula; OPN, osteopontin; siRNA, small interfering RNA.

and ER $\alpha$ may be involved in LWDHF-mediated upregulation of $\alpha$-SMA.

\section{Discussion}

Our previous study investigated the effects of LWDHF on ovariectomized rats with atherosclerosis and demonstrated that LWDHF improved lipid metabolism in the serum and reduced Ang II levels through regulating ER expression, thereby producing therapeutic effects (15). In addition, our in vitro study demonstrated that LWDHF-medicated serum protected HUVECs against $\mathrm{H}_{2} \mathrm{O}_{2}$-induced apoptosis via $\mathrm{ER} \alpha$ (16). Therefore, it may be hypothesized that LWDHF exerts preventive and therapeutic effects against atherosclerosis in menopausal women, and that ERs may mediate the effects of LWDHF. The present study provided further evidence supporting the antiatherosclerotic effects of LWDHF, indicating that LWDHF inhibited the Ang II-induced phenotypic modulation and migration of VSMCs, and that the effects of LWDHF were associated with ERs, particularly ER $\alpha$.

The development of atherosclerosis- and restenosis-associated advanced lesions is highly dependent on VSMC proliferation. Therefore, inhibition of VSMC proliferation is considered a potential strategy in the prevention of atherosclerosis (7). LWDH is a commonly used traditional Chinese formula; the present study demonstrated that LWDH could inhibit Ang II-induced VSMC proliferation in a dose-dependent manner, and the maximum inhibition on proliferation was detected following treatment with $12 \mu \mathrm{g} / \mathrm{ml} \mathrm{LWDH} \mathrm{(73.24 \% ).}$ It is well known that progression from $\mathrm{G}_{1}$ to $\mathrm{S}$ phase is closely associated with cell proliferation. The present study provided further evidence to confirm that LWDHF may exert antiproliferative effects; Ang II-induced cell cycle progression to S phase was markedly reduced by $12 \mu \mathrm{g} / \mathrm{ml}$ LWDHF, as determined by flow cytometry. Migration also has an important role in numerous vascular diseases. In the present study, LWDHF exerted marked inhibitory effects on VSMC migration, as determined using wound-healing and Transwell chamber assays. Cell migration is associated with numerous spatially and temporally coordinated cellular processes, and occurs in the following four steps: i) Formation of actin-rich protrusions, such as lamellipodia, ii) cell adhesion, iii) translocation of the cell body and iv) rear detachment. Cytoskeletal reorganization is required for leading-edge cellular protrusion during locomotion, and VSMC migration requires rearrangement in the VSMC cytoskeleton $(26,27)$. Consistent with previous studies, the present study demonstrated that treatment with LWDHF could markedly suppress Ang II-stimulated rearrangement of F-actin.

Phenotypic modulation of VSMCs is the cytological basis for vascular remodeling. Under normal conditions, VSMCs with a contractile phenotype are highly specialized cells, which function to regulate blood vessel diameter, blood pressure and blood flow distribution. However, VSMCs exhibit a phenotypic alteration, characterized by loss of contractility, and abnormal proliferation and migration, in response to vascular injury or disease. The present study hypothesized that LWDHF may inhibit proliferation and migration of VSMCs via modulating the VSMC phenotype. In general, $\alpha$-SMA is considered a biomarker of VSMCs with a contractile phenotype, whereas OPN is a biomarker of VSMCs with a synthetic phenotype $(28,29)$. VSMC phenotypic switching is characterized by markedly increased expression of synthetic phenotype markers, alongside increased VSMC proliferation and migration. Consistent with the previous observations, treatment of VSMCs with Ang II resulted in downregulation of $\alpha$-SMA and upregulation of OPN; these effects were suppressed by LWDHF in a concentration-dependent manner. These results confirmed the hypothesis that LWDHF may inhibit proliferation and migration of VSMCs via modulating the VSMC phenotype. However, the addition of tamoxifen to the culture medium partly abolished the inhibitory effects of LWDHF on phenotypic switching, thus suggesting that ERs may be involved in the effects of LWDHF on VSMC phenotype.

A previous study demonstrated that LWDHF may possess estrogenic properties and regulate the expression of ERs (16). The present study demonstrated that LWHDF could suppress VSMC proliferation in vitro. To further investigate the association between LWHDF and ER, western blot analysis and RT-qPCR were employed to examine the effects of LWDHF on ER $\alpha$ and ER $\beta$ expression. The results indicated that LWDHF upregulated the expression levels of ERs, particularly ER $\alpha$. The underlying mechanism of inhibitory actions with LWDHF on VSMCs has been intensely investigated following the discovery of ER $\alpha$. Our earlier studies in HUVECs indicated that LWDHF 
exerted anti-apoptotic effects, mainly via an ER $\alpha$-mediated estrogen signaling pathway (16). Therefore, to determine whether $\mathrm{ER} \alpha$ mediated the inhibitory effects of LWDHF on phenotypic modulation of VSMCs, siRNA technology was used to reduce the expression of ER $\alpha$ in HUVSMCs, which led to a significant increase in the Ang II-induced synthetic phenotype. In addition, $\mathrm{ER} \alpha$ knockdown in HUVSMCs significantly attenuated the effects of LWDHF on phenotypic modulation. LWDHF-elevated $\alpha$-SMA expression was markedly reduced in HUVSMCs treated with ER $\alpha$ siRNA, whereas OPN expression was not markedly altered in ER $\alpha$ siRNA-transfected cells treated with Ang II and LWDHF compared with in control siRNA-transfected cells. These data suggested that LWDHF may upregulate $\alpha$-SMA expression predominantly via ER $\alpha$-mediated signaling pathways; however, another ER subtype may be responsible for LWDHF-induced downregulation of OPN.

In conclusion, the present study suggested that LWDHF possesses estrogenic properties and may mimic E2 to suppress VSMC proliferation and migration in vitro. The effects exerted by LWDHF were associated with phenotypic regulation. In addition, analysis of the underlying mechanisms of action revealed that ER $\alpha$ may act as a key mediator for LWDHF-induced inhibition of phenotypic switching. However, whether ER $\beta$ is involved in the inhibitory effects of LWDHF on phenotypic switching and migration of VSMCs requires further research.

\section{Acknowledgements}

The present study was supported by the National Natural Science Foundation of China (grant no. 81774029).

\section{Competing interests}

The authors declare that they have no competing interests.

\section{References}

1. Rivard A and Andrés V: Vascular smooth muscle cell proliferation in the pathogenesis of atherosclerotic cardiovascular diseases. Histol Histopathol 15: 557-571, 2000.

2. Lacolley P, Regnault V, Nicoletti A, Li Z and Michel JB: The vascular smooth muscle cell in arterial pathology: a cell that can take on multiple roles. Cardiovasc Res 95: 194-204, 2012.

3. Deatrick KB, Eliason JL, Lynch EM, Moore AJ, Dewyer NA, Varma MR, Pearce CG, Upchurch GR Jr, Wakefield TW and Henke PK: Vein wall remodeling after deep vein thrombosis involves matrix metalloproteinases and late fibrosis in a mouse model. J Vasc Surg 42: 140-148, 2005.

4. Owens GK, Kumar MS and Wamhoff BR: Molecular regulation of vascular smooth muscle cell differentiation in development and disease. Physiol Rev 84: 767-801, 2004.

5. Rensen SS, Doevendans PA and van Eys GJ: Regulation and characteristics of vascular smooth muscle cell phenotypic diversity. Neth Heart J 15: 100-108, 2007.

6. Schwartz SM: Perspectives series: cell adhesion in vascular biology. Smooth muscle migration in atherosclerosis and restenosis. J Clin Invest 99: 2814-2816, 1997.

7. Doran AC, Meller N and McNamara CA: Role of smooth muscle cells in the initiation and early progression of atherosclerosis. Arterioscler Thromb Vasc Biol 28: 812-819, 2008.

8. Hao H, Gabbiani G and Bochaton-Piallat ML: Arterial smooth muscle cell heterogeneity: implications for atherosclerosis and restenosis development. Arterioscler Thromb Vasc Biol 23: 1510-1520, 2003.
9. Babapulle MN and Eisenberg MJ: Coated stents for the prevention of restenosis: Part I. Circulation 106: 2734-2740, 2002.

10. Zhao Y, Liu YX, Xie SL, Deng BQ, Wang JF and Nie RQ: Increased expression of granulocyte colony-stimulating factor mediates mesenchymal stem cells recruitment after vascular injury. Chin Med J (Engl) 124: 4286-4292, 2011.

11. Watanabe T, Pakala R, Katagiri T and Benedict CR: Synergistic effect of urotensin II with mildly oxidized LDL on DNA synthesis in vascular smooth muscle cells. Circulation 104: 16-18, 2001.

12. Intengan HD and Schiffrin EL: Vascular remodeling in hypertension: roles of apoptosis, inflammation, and fibrosis. Hypertension 38: 581-587, 2001.

13. Liu JP, Feng L, Zhang MH, Ma DY, Wang SY, Gu J, Fu Q, Qu R and Ma SP: Neuroprotective effect of Liuwei Dihuang decoction on cognition deficits of diabetic encephalopathy in streptozotocin-induced diabetic rat. J Ethnopharmacol 150: 371-381, 2013.

14. Kalu DN: The ovariectomized rat model of postmenopausal bone loss. Bone Miner 15: 175-191, 1991.

15. Wu Y, L Y and Zhang QC: The effect of LWDHF on menopausal atherosclerosis model in ovariectomized rats. Chinese Traditional Patent Medicine 34: 553-556, 2012 (In Chinese).

16. Yin QY, Guo J and Meng QH: Effects of Liuweidihuang formula mediated serum on $\mathrm{H}_{2} \mathrm{O}_{2}$-injured human umbilical vascular endothelial cells. Chin Pharmacol Bull 29: 1753-1757, 2013 (In Chinese).

17. Mendelsohn ME and Karas RH: Molecular and cellular basis of cardiovascular gender differences. Science 308: 1583-1587, 2005.

18. Farhat MY, Lavigne MC and Ramwell PW: The vascular protective effects of estrogen. FASEB J 10: 615-624, 1996.

19. Kawagoe J, Ohmichi M, Tsutsumi S, Ohta T, Takahashi K and Kurachi H: Mechanism of the divergent effects of estrogen on the cell proliferation of human umbilical endothelial versus aortic smooth muscle cells. Endocrinology 148: 6092-6099, 2007.

20. Sivritas D, Becher MU, Ebrahimian T, Arfa O, Rapp S, Bohner A, Mueller CF, Umemura T, Wassmann S, Nickenig G, et al: Antiproliferative effect of estrogen in vascular smooth muscle cells is mediated by Kruppel-like factor- 4 and manganese superoxide dismutase. Basic Res Cardiol 106: 563-575, 2011.

21. Liu HM, Zhao XF, Guo LN, Tan Z and Wang TH: Effects of caveolin-1 on the $17 \beta$-estradiol-mediated inhibition of VSMC proliferation induced by vascular injury. Life Sci 80 : 800-812, 2007.

22. Yang S, Zhou W, Zhang Y, Yan C and Zhao Y: Effects of Liuwei Dihuang decoction on ion channels and synaptic transmission in cultured hippocampal neuron of rat. J Ethnopharmacol 106: 166-172, 2006.

23. Rodríguez A, Fortuño A, Gómez-Ambrosi J, Zalba G, Díez J and Frühbeck G: The inhibitory effect of leptin on angiotensin II-induced vasoconstriction in vascular smooth muscle cells is mediated via a nitric oxide-dependent mechanism. Endocrinology 148: 324-331, 2007.

24. Livak KJ and Schmittgen TD: Analysis of relative gene expression data using real-time quantitative PCR and the 2(-Delta Delta C(T)) Method. Methods 25: 402-408, 2001.

25. Hedin U and Thyberg J: Plasma fibronectin promotes modulation of arterial smooth-muscle cells from contractile to synthetic phenotype. Differentiation 33: 239-246, 1987

26. Rousseau S, Houle F, Landry J and Huot J: p38 MAP kinase activation by vascular endothelial growth factor mediates actin reorganization and cell migration in human endothelial cells. Oncogene 15: 2169-2177, 1997.

27. Gunst SJ and Zhang W: Actin cytoskeletal dynamics in smooth muscle: a new paradigm for the regulation of smooth muscle contraction. Am J Physiol Cell Physiol 295: C576-C587, 2008.

28. Shanahan CM, Weissberg PL and Metcalfe JC: Isolation of gene markers of differentiated and proliferating vascular smooth muscle cells. Circ Res 73: 193-204, 1993.

29. Hultgårdh-Nilsson A, Lövdahl C, Blomgren K, Kallin B and Thyberg J: Expression of phenotype-and proliferation-related genes in rat aortic smooth muscle cells in primary culture. Cardiovascular research 34: 418-430, 1997.

This work is licensed under a Creative Commons Attribution-NonCommercial-NoDerivatives 4.0 International (CC BY-NC-ND 4.0) License. 